\title{
BOUND AND RESONANT STATES OF SHALLOW DONORS IN QUANTUM ANTIDOTS
}

\author{
R. Buczko \\ Institute of Physics, Polish Academy of Sciences \\ Al. Lotników 32/46, 02-668 Warszawa, Poland \\ AND F. BASSANI
}

Scuola Normale Superiore, Piazza dei Cavalieri, 56100 Pisa, Italy

\begin{abstract}
The energy spectrum and the wave functions of a shallow donor placed at the center of a spherical quantum antidot are computed within the effective mass approximation. The wave functions for discrete bound states and for continuum states are obtained in a closed form. We show that, due to the local potential of the microstructure, resonances occur in the continuum. Their energies are close to those of hydrogen-like levels lying under the top of the barrier when the quantum antidot radius is large as compared to the effective Bohr radius. The lifetimes of the resonant states and the oscillator strengths for optical transitions from the ground state are computed. We show how the energy spectrum and the oscillator strengths depend on the antidot parameters.
\end{abstract}

PACS numbers: 73.20.Dx, 78.66.-w, 71.55.-i

Zero-dimensional microstructures, known as quantum dots (QDs) confine electrons and (or) holes inside a small region. The lack of translational symmetry produces atom-like discrete levels, whose energies can be varied just by changing the QD diameter. Like for real atoms, one can expect to obtain information about these energy levels by studying the optical transitions. In a previous paper [1] we have presented theoretical results of electron states in a spherical QD. We have shown that $\mathrm{QD}$ structures produce not only discrete levels but also resonant states in the continuum. Their positions and energy widths depend on the well size and on the values of the confining potential. There is a correspondence between bound and resonant states. As the radius of the QD decreases, the bound states continuously transform into resonant states and a critical value of the radius for this transition can be found for each state.

The Coulomb potential alone does not produce any resonances in the continuum. However, if it is complemented with the potential of the dot, resonant states appear. In the case of superimposed Coulomb repulsive potential (which can be given, for example, by a compensated acceptor), the resonances are very narrow 
and are of the type considered by Breit and Wigner [2] for $\alpha$ decay in nuclear physics. In the case of the Coulomb attractive potential of a donor inside the dot the resonances are much wider. But they still play an important role in the interband optical transitions. For example: with decreasing dot radius the oscillator strength for the transition from the ground to bound excited states falls down to almost zero and is transferred to the continuum when the first $p$-type resonant state appears.

In this paper we study another possibility, when superposition of the potentials of the donor and the zero-dimensional structure leads to appearance of resonant states. This is the case of a donor inside an antidot. As in the previous paper [1], we use the effective mass and spherical approximations and carry out the calculations of the continuum states within standard quantum mechanics scattering theory. The analytical solutions of the effective mass equation inside and outside the quantum antidot (QAD) are given in terms of spherical harmonics and Kummer functions. The QAD potential is given by: $U(r)=U_{0}$, for $r<R$ and $U(r)=0$, for $r>R$, where $R$ is the antidot radius. The appropriate boundary conditions give the discrete levels and the solutions in the continuum as well. The resonant state energies are obtained from the phase shift between incoming and outgoing spherical waves. We use the effective rydberg $\left(\mathrm{Ry}^{*}\right)$ and the effective Bohr radius $\left(a^{*}\right)$ as the energy and length units.

In Fig. 1 we present the dependence of the energies of the states on the QAD radius $(R)$ for the potential barrier equal to $3 R y^{*}$. As can be seen for very small $R$ the energies of the bound states are very close to the hydrogen-like ones, because the potential of QAD corresponds only to a repulsive central-cell correction to the impurity potential. When the radius increases the bound state, wave functions are pushed away from the center by the antidot potential and the binding energies diminish to zero for a very large QAD. At the same time resonances in the continuum appear. Their energies fall down at the beginning. When the radius is increased

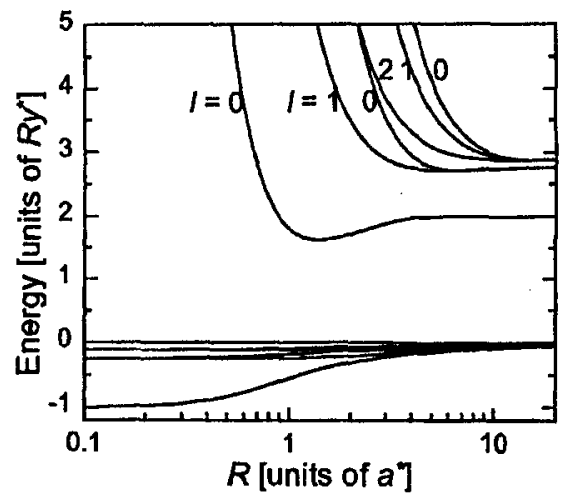

Fig. 1. Energies of donor states in spherical QAD with a potential barrier $U_{0}=3 R y^{*}$. The lower states correspond to the total momentum quantum number $l=0,1,2$. The energy values $E>0$ correspond to resonant states in the continuum. 


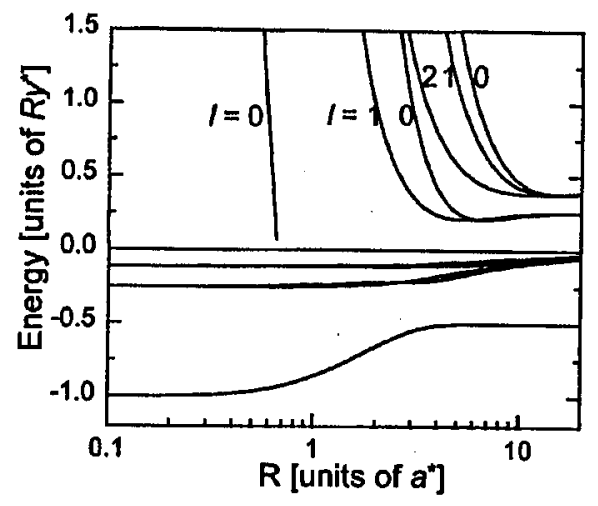

Fig. 2. The same as in Fig. 1, but for QAD potential barrier $U_{0}=(1 / 2) \mathrm{Ry}^{*}$.

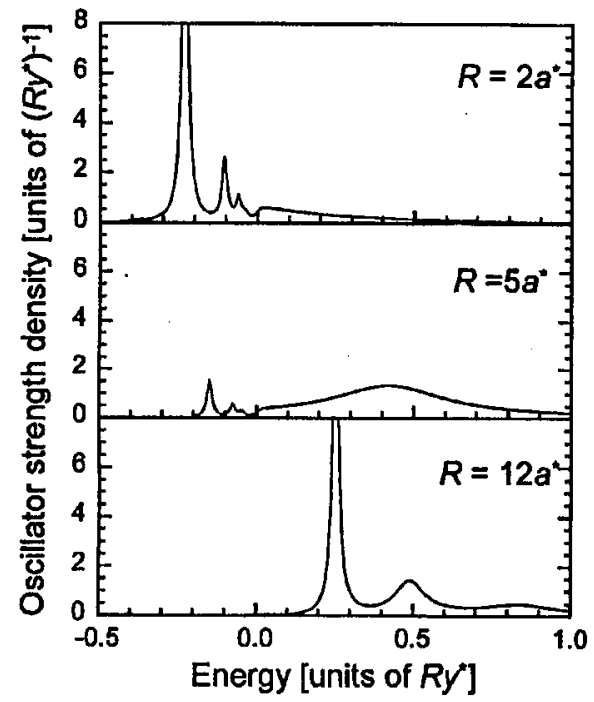

Fig. 3. Computed spectra of transitions from the ground state $(l=0)$ to the excited $p$-type bound and continuum states of the QAD with $U_{0}=(1 / 2) \mathrm{Ry}^{*}$. Results are presented for three different antidot radii. The energies refer to the ionization limit.

to the value about $1 a^{*}$, the energy of the first $s$-type $(l=0)$ resonant state reaches the minimum value and then it increases tending to the energy value of $U_{0}-1 R y^{*}$, which corresponds to the hydrogen-like binding energy below the top of the QAD potential. Also the other resonant states acquire the hydrogen-like $U_{0}-1 / n^{2} \mathrm{Ry}^{*}$ energies below the top of the QAD barrier for large radii. We have checked that the widths of the resonances diminish to very small values when $R>10 a^{*}$. Therefore, we can define them as hydrogen-like states in the large $r$ limit. The wave functions of the bound states and the localized part of the wave functions of the resonant ones lie in the separate space regions. Hence the cross-sections for opti- 
cal transitions from the ground to the resonant states are close to zero. In order to obtain a bigger transition probability the QAD potential must be lower than 1Ry*. In Fig. 2 we present the results for $U_{0}=(1 / 2) R y^{*}$. We can see that in this case the energy of the lowest $s$-type resonance falls down below 0 , that is in the region of bound states. In the intermediate radius region the ground state wave function is localized partially inside and partially outside the antidot. For large radius, the ground state energy tends to the value of $-(1 / 2) \mathrm{Ry}^{*}$ and the ground state wave function is totally localized inside the dot. This behavior influences the optical properties of QAD. We present in Fig. 3 the calculated oscillator strengths of optical transitions for three different QAD radii. For $R=2 a^{*}$ we observe distinct transitions to bound states and rather weak transitions to the continuum. For $R=5 a^{*}$ the transitions to the continuum are much stronger and for $R=12 a^{*}$ all the oscillator strength goes to the well defined hydrogen-like resonances. The presented case of the antidot with the small potential barrier can be realized within the GaAs/ $\mathrm{Ga}_{1-x} \mathrm{Al}_{x} \mathrm{As}$ structure for a very small Al composition $x=0.33 \%$.

Similarly to the case of a donor inside QD, we can distinguish two asymptotic regions (small and large antidot radii) with hydrogen-like states. Irowever, in QAD the states in the barrier are rosonant and there is no correspondence between bound and resonant states. Only if the barrier is small enough $\left(U_{0}<1 R y^{*}\right)$ and the antidot radius is equal to a few effective Bohr radii, we can observe optical transitions to both type of states.

\section{References}

[1] R. Buczko, F. Bassani, Phys. Rev. B 54, 2667 (1996).

[2] E. Wigner, Phys. Rev. 98, 145 (1955); G. Breit, Phys. Rev. 107, 1612 (1957). 